Orthopäde $2009 \cdot 38: 483-483$

DOI 10.1007/s00132-009-1410-1

(C) Springer Medizin Verlag 2009

\author{
V. Krenn ${ }^{1} \cdot$ W. Rüther ${ }^{2}$ \\ ${ }^{1}$ Zentrum für Histologie, Zytologie und Molekulare Diagnostik Trier \\ ${ }^{2}$ Klinik und Poliklinik für Orthopädie, Universitätsklinikum Hamburg-Eppendorf, \\ Klinikum Bad Bramstedt, Hamburg
}

\title{
Orthopädische Pathologie
}

In der Deutschen Gesellschaft für Pathologie wurde im letzten Jahr der Arbeitskreis „Orthopädische Pathologie“ gegründet. Hiermit wird das Ziel verfolgt, größere Aufmerksamkeit auf die Pathologie des Bewegungssystems zu lenken.

Für den Orthopäden hat die histopathologische Diagnostik in den meisten Fällen eine eher untergeordnete Bedeutung. Tatsächlich kommt der diagnostischen Relevanz der histopathologischen Untersuchungsbefunde eine sehr variable Wertigkeit zu, die von der rein bestätigenden bis zur erkrankungsdefinierenden Diagnostik reicht.

In den letzten Jahren wurden durch systematische Analysen neue histopathologische Typisierungen und Klassifikationen entwickelt. Diese betreffen die Diagnostik degenerativer, entzündlicher und infektiöser Krankheiten. Hieraus resultierende diagnostische Sicherheit kann zu unmittelbaren Konsequenzen für den klinisch tätigen Orthopäden führen.

Die Diagnostik der bakteriellen Infektion ist keineswegs eine Domäne der Mikrobiologie. Die Zuverlässigkeit der histologischen Diagnostik steht der mikrobiologischen keineswegs nach. Die Diagnostik von minimalen bakteriellen Infektionen (sog. Low-grade-Infektionen) sind ebenso wie die falsch-positiven mikrobiologischen Befunde ein Problem, das sich durch zusätzliche, histopathologisch definierte Kriterien lösen lässt. Mit neueren molekularbiologischen Methoden kann die Infektion abgesichert werden.

Das differentialdiagnostische Spektrum der Synovialitis ist vielfältig. Durch einen histopathologischen Synovialitisscore erfolgt eine Stratifizierung in Lowund High-grade-Synovialitiden, die eine Zuordnung zu degenerativen und ent- zündlichen Gelenkerkrankungen ermöglichen. Sogenannte Pseudogichtarthropathien (Pyrophosphate, Apatite - u. a. Kalziumphasphatkristalle) sind häufiger überraschende Befunde, die bei fortgeschrittenen degenerativen Arthropathien sogar die Regel sind. Die Bedeutung der Chondrokalzinose für die Behandlung einer Arthropathie findet allerdings aktuell wenig Beachtung.

Die degenerative Arthropathie (Osteoarthrose) stellt die häufigste Krankheit im fortgeschrittenen Lebensalter dar. Die histopathologische Untersuchung exzidierten Gewebes, das z. B. während einer Endoprothesenoperation gewonnen wurde, wird in aller Regel die bekannte Diagnose bestätigen. Zu Recht wird die Frage gestellt, ob die histopathologische Diagnostik nach wie vor notwendig ist, zumal sie bei der Untersuchung von Knochen aufwändig ist.

Die Ursachen der Endoprothesenlockerung sind uneinheitlich. Das von Krenn et al. vorgeschlagene Klassifikationsschema ermöglicht eine Aussage über die Ursache der Prothesenlockerung, die möglicherweise für den Kliniker therapeutische Konsequenzen hat.

Die Neoplasien des Bewegungssystems stellen eine heterogene Gruppe mit unterschiedlich malignem Potential dar. Knochentumoren waren schon immer eine interdisziplinäre Herausforderung, in der Spezialisten gefordert sind. Die vorgestellte Übersichtsarbeit verweist einmal mehr auf dieses Problem hin - schon vor der Biopsie ist die Kompetenz eines interdisziplinären Gremiums gefragt.

Die in diesem Heft zusammengetragenen Übersichten sollen den Stellenwert der Histopathologie unter modernen Aspekten beleuchten.
Der Histopathologie sollte es v. a. mit molekular-pathologischen Methoden gelingen, auch bei der breiten Palette der sog. degenerativen Krankheiten zu einer krankheitsdefinierenden Diagnostik beizutragen. Zumindest wäre eine Entwicklung in diese Richtung wünschenswert.

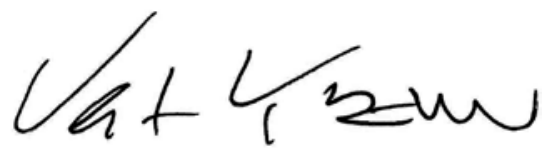

V. Krenn

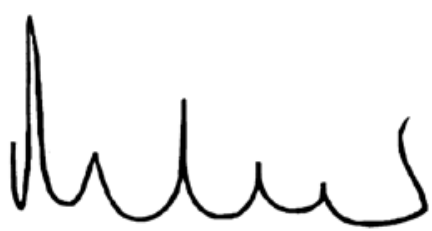

W. Rüther

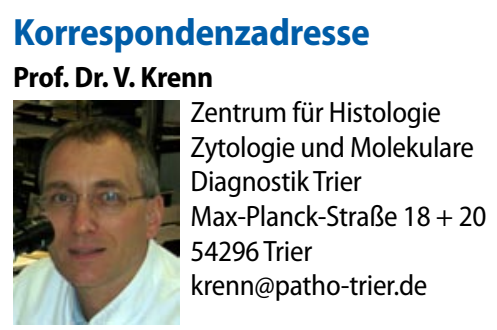

Prof. Dr. W. Rüther

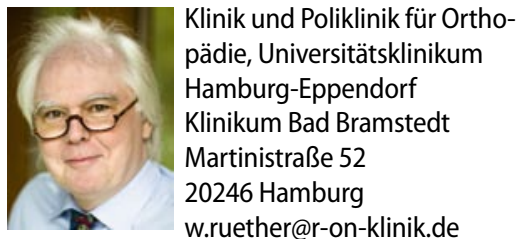

